\title{
Ethyl 2-(Diisopropoxyphosphoryl)-2H-Azirine-3-Carboxylate: Reactions with Activated 1,3-Butadienes
}

M. José Alves, ${ }^{[\mathrm{a}] *}$ Américo Lemos, ${ }^{[\mathrm{b}]}$ José Enrique Rodriguez-Borges,${ }^{[\mathrm{c}]}$ and Xerardo García-Mera $^{[\mathrm{d}]}$

[a] Departamento de Química, Universidade do Minho, Campus de Gualtar, 4710-057 Braga, Portugal. ${ }^{[b]}$ Departamento de Química, Bioquímica e Farmácia, Universidade do Algarve, Campus de Gambelas, 8005-137 Faro, Portugal. ${ }^{[c]}$ CIQ, Departamento de Química, Faculdade de Ciências, Universidade do Porto, Rua do Campo Alegre, 687, 4169007 Porto, Portugal. [d] Departamento de Química Orgánica, Facultade de Farmacia, Universidade de Santiago de Compostela, E-15782 Santiago de Compostela, Spain

\footnotetext{
Abstract: Ethyl 2-(diisopropoxyphosphoryl)-2H-azirine-3-carboxylate was generated "in situ" and reacted with a number of 1,3-butadienes. Cycloadducts, or in one case an ensuing rearranged product, were isolated with moderate to good yields.
}

Keywords: $2 H$-Azirines, aza-Diels-Alder cycloaddition, dienophiles, phosphonate derivative compounds.

* Corresponding author. Fax: +00-351-253-678983. e-mail: mja@quimica.uminho.pt 


\section{Introduction}

$2 \mathrm{H}$-Azirines have generated a great deal of interest due to its versatility as building blocks in the synthesis of important classes of heterocyclic compounds, ${ }^{1,2}$ and amino acids. ${ }^{3} 2 H_{-}$ Azirines bearing ester groups are especially important not only due to its structural similarity to natural occurring azirines with biological activity, like azirinomycin ${ }^{4}$ and $(R)$ (-)-dysidazirine antibiotics, ${ }^{5}$ but also for being excellent precursors at the synthesis of $\alpha_{-}{ }^{6}$ and $\beta$-aminoacid ${ }^{7}$ derivatives. Azirines with $\mathrm{C}=\mathrm{O}, \mathrm{P}=\mathrm{O}$ or heteroaromatic groups conjugated with the $\mathrm{C}=\mathrm{N}$ bond, are effective dienophile partners ${ }^{8-10}$ in Diels-Alder cycloadditions, producing bicyclic and tricyclic compounds. In the absence of such conjugation they are much less reactive turning them useless in most cases. There are only few examples of reactions of simple azirines to especially activated 1,3diphenylisobenzofuran occurring under high temperature ${ }^{11 \mathrm{a}, \mathrm{b}}$ or Lewis acid catalysis ${ }^{11 \mathrm{c}, \mathrm{d}}$ but they are exceptions.

As we recently found, ${ }^{12}$ that Diels-Alder cycloadducts derived from 2,3-bifunctionalized azirines and Danishefsky diene (1), evolve to 1,2-di-substituted-pyridin-4-ones 2, a very interesting type of compounds (Scheme 1) we seek for the synthesis of azirines capable of entering in such type of reactions.
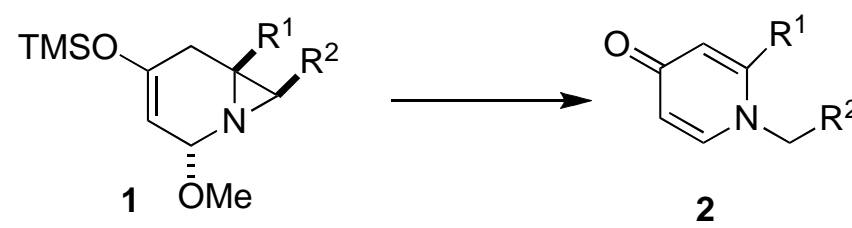

$$
\begin{aligned}
& \mathrm{R}^{1}=\mathrm{CO}_{2} \mathrm{Me} ; \mathrm{R}^{2}=2,6 \text {-dichlorophenyl } \\
& \mathrm{R}^{1}=\mathrm{CO}_{2} \mathrm{Me} ; \mathrm{R}^{2}=\text { tolyl } \\
& \mathrm{R}^{1}=2-\mathrm{Py} ; \mathrm{R}^{2}=\mathrm{CO}_{2} \mathrm{Et}
\end{aligned}
$$

Scheme 1: 1,2-Di-substituted pyridin-ones 2 obtained from cycloadducts 1

Figure 1 represents a few examples of such azirines known: 3-(2-pyridyl)-2-oxycarbonyl azirine $\mathbf{I}^{10}{ }^{10}$ the highly reactive di-ester azirine $\mathbf{I I},{ }^{13}$ and 3-(3-benzofuryl)-2-oxycarbonyl azirine III. ${ }^{14}$ There are a few more azirines with an extra substitution at C-2. According to early results in our group 2,2-disubstituted azirines would be non reactive, ${ }^{15}$ possibly for steric reasons. 3-Alcoxyphosphoryl-2-trifluormethyl-2 $H$-azirine-2-carboxylate has been recently reported, as marginal side product, ${ }^{16}$ but it would be equally non reactive. On the other hand, 2-alcoxyphosphoryl-2H-azirine-3-carboxylates would be excellent candidates for such reactions and would introduce a biological interesting phosphonate group in 
products. ${ }^{17}$ As far as we know, such compounds had never been produced, regardless to be closely related to $\alpha$-amino- $\beta$-phosphonic carboxylic compound ( $S$ )-AP-3, known to be modulator for the $N$-methyl-D-aspartate (NMDA) receptor site. (Figure 2) Compounds of such type are the most common neurotransmitters in the mammalian central nervous system that have been exploited in the treatment of several pathological conditions affecting brain, such as Parkinson's and Alzheimer's diseases. ${ }^{18}$<smiles>COC(=O)C1=NC1C1=NC1c1ccccn1</smiles>

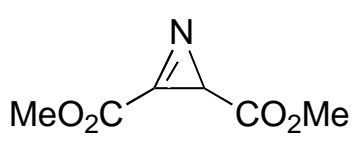

III<smiles>COC(=O)C1=NC1c1cc2ccccc2o1</smiles>

Figure 1: 2,3-Di-substituted $2 \mathrm{H}$-azirines bearing a conjugated electron withdrawing group at 3-position.<smiles>NC(CP(=O)(O)O)C(=O)O</smiles>

Figure 2:(S)-2-Amino-3-phosphonopropanoic acid modulator of NMDA.

Compounds of type $\mathbf{2}$ can be regarded as precursors of iminosugars. Initial reduction of the ketone function in compound 2 , in the presence of a chiral ligand such as (-)- $N-$ methylephedrine would lead to a chiral alcohol, from which upon Sharpless epoxidation iminosugar structures can be deduced. Ganem successfully obtained a chiral alcohol intermediate in the synthesis of an isofagomine derivative, from a $\alpha, \beta$-insaturated ketone integrated in a piperidine structure. ${ }^{19}$

In this paper we report the unprecedented generation of 2-dialkoxyphosphoryl-2 $H$-azirine3 -carboxylate and its interception by a number of electron rich 1,3-butadienes, including Danishefsky diene. 


\section{Results and Discussion}

The oxime 4 was first obtained from ethyl bromopyruvate oxime $\mathbf{3}^{20}$ and triisopropylphosphite. Its treatment with tosyl chloride in the presence of $\mathrm{Na}_{2} \mathrm{CO}_{3}$ led to $\beta$-phosphonic tosyloxime ester 5. (Scheme 2) The corresponding $2 H$-azirine 6 was obtained under Neber conditions, but could not be isolated from the reaction medium. Monofunctional 2-phosphonic azirine esters ${ }^{21}$ had been produced and isolated before, under similar reaction conditions. In the present case however manipulation of the reaction mixture led to decomposition, according to ${ }^{1} \mathrm{H}$ NMR analysis.

In a typical procedure tosyloxime 5 was solubilized in benzene mixed with $\mathrm{K}_{2} \mathrm{CO}_{3}$ (10 eq.), triethylamine ( 0.3 eq.) and 1,3-butadiene, and stirred for 4 days at room temperature. Three primary cycloadducts 7 were obtained in $9.3-59.4 \%$ yield. Derivative 8 was obtained in the case of reaction with Danishefsky diene in $38.5 \%$ yield, by rearrangement of the primary cycloadduct 7c. (Scheme 3 )

Yields were moderated to good with one exception. Cycloadduct obtained from reaction with 2,3-dimethyl 1,3-butadiene was isolated in low yield, even in the presence of excess of diene. (5 eq.) Davis ${ }^{9}$ also found difficulties in reaction of a 3 -phosphonate $2 H$-azirine with 2,3-dimethyl 1,3-butadiene reporting the need of 100 equivalents of the diene.

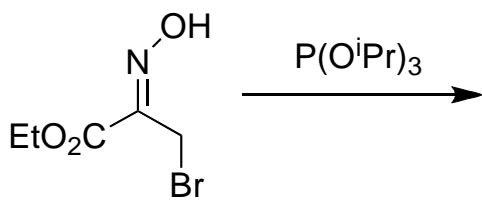

3<smiles>CCCOP(=O)(OCC)/C(CC(=O)OCC)=N/O</smiles>

4
$\mathrm{TsC}$

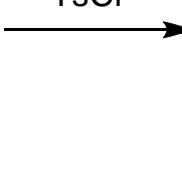

Oipr

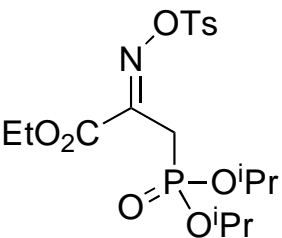

5

Scheme 2: Synthesis of tosyloxime $\mathbf{5}$ 


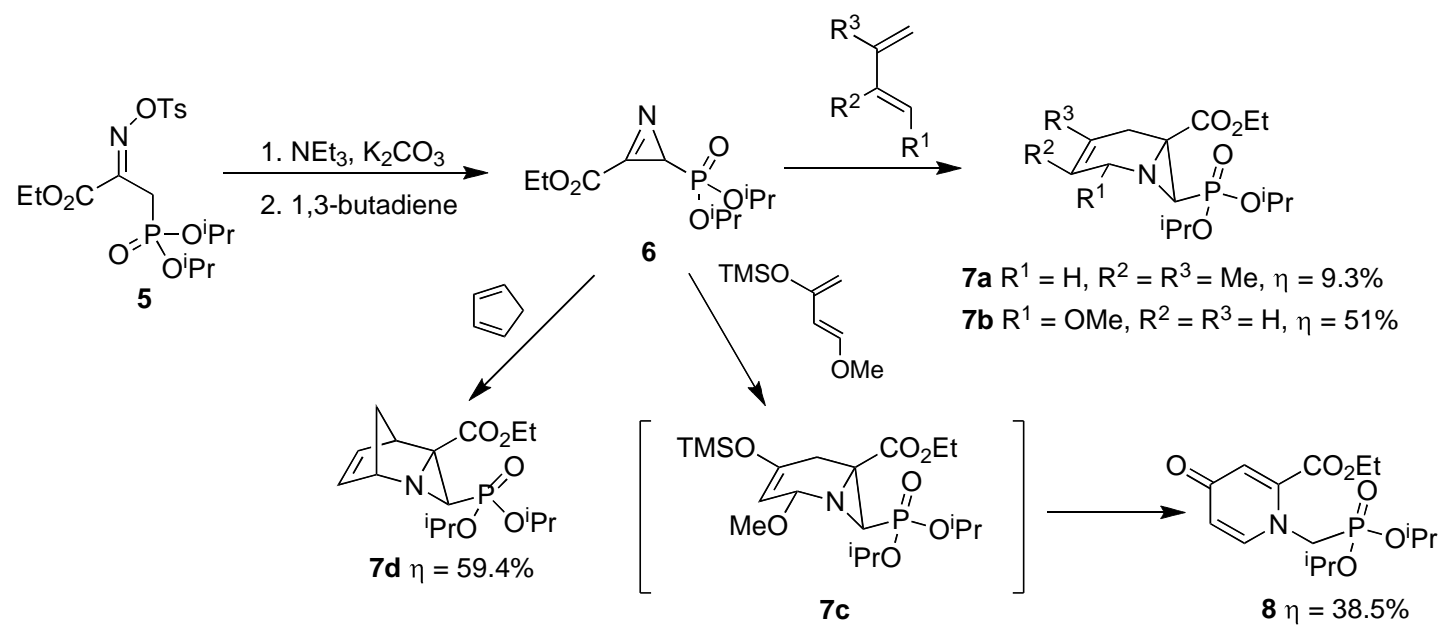

Scheme 3: Diels-Alder cycloaddition of ethyl 2-(diisopropoxyphosphoryl)-2H-azirine-3-carboxylate to 1,3-butadienes

Reaction of the azirine 6 with 1-metoxy-1,3-butadiene evidenced the regioselectivity of cycloaddition to be governed by primary electronic effects. ${ }^{1} \mathrm{H}$ and ${ }^{13} \mathrm{C}$ NMR spectra data of product $\mathbf{7 b}$ showed to be in accordance with the electron-withdrawing effect of the two heteroatoms attached to C-2: $\mathrm{H}-2$ showed up at $\delta_{\mathrm{H}}=4.80 \mathrm{ppm}$ and $\mathrm{C}-2$ at $\delta_{\mathrm{C}}=85.6 \mathrm{ppm}$. The cycloaddition products were obtained as single isomers and estimated to be formed by endo kinetic processes, as generally occurs in reactions of $2 \mathrm{H}$-azirines with 1,3 -dienes. ${ }^{8}$ Furan and their derivatives are exceptions due to retro-Diels-Alder cycloadditions of the initially formed kinetic endo cycloadduct that isomerize to the thermodynamic exo products. ${ }^{11 \mathrm{~b}}$ The low field resonance of $\mathrm{H}-3$ is a feature of endo cycloadducts of azirines to cyclopentadiene. ${ }^{8}$ This can be ascribed to the anisotropy of the backside double bond over $\mathrm{H}-3$ due to constrain of the tricyclic structure. The chemical shift value of $\mathrm{H}-3$ in compound $7 \mathbf{d}$ correspond to such effect appearing at $\delta_{\mathrm{H}}=1.62 \mathrm{ppm}$.

\section{Conclusion}

Cycloaddition of ethyl 2-(diisopropoxyphosphoryl)-2H-azirine-3-carboxylate to nucleophilic dienes gave a number of functionalized six-membered ring fused aziridines to which applications to the synthesis of interesting biological compounds can be envisaged. Development of asymmetric cyclization of the azirine is ongoing. 


\section{Experimental Section}

\section{General Methods}

${ }^{1} \mathrm{H}$ NMR spectra and ${ }^{13} \mathrm{C}$ NMR spectra $(75.47 \mathrm{MHz})$ were recorded on a Varian Unity Plus $300(300 \mathrm{MHz})$ spectrometer or on a Bruker WM AMX (300 MHz) spectrometer or on a Bruker Avance $\mathrm{II}^{+}(400 \mathrm{MHz})$ using TMS as internal standard (chemical shifts $(\delta)$ in ppm, $J$ in Hz). Multiplicities are recorded as broad peaks (br), singlets (s), doublets (d), triplets $(\mathrm{t})$, doublets of doublets $(\mathrm{dd})$, doublets of triplets $(\mathrm{dt})$, triplets $(\mathrm{t})$, quartets $(\mathrm{q})$ and multiplets (m). $J$ values are in $\mathrm{Hz}$ and $\delta$ in ppm. Infrared spectra were recorded on a Perkin-Elmer 1640-FT spectrophotometer and the main bands are given in $\mathrm{cm}^{-1}$. Samples were run as thin films. Mass Spectra were recorded on a VG Autospec M. Purification of crude samples was done by dry flash chromatography, using silica gel purchased from Carlo Erba (35-70 m $\mu$ ).

\section{Synthesis of ethyl 3-diisopropoxyphosphoryl-2-hydroxyiminopropanoate 4}

To ethyl bromopyruvate oxime ${ }^{19}(4.7 \mathrm{~g}, 22 \mathrm{mmol})$ dissolved in dichloromethane $(30 \mathrm{~mL})$ was added tri-isopropylphosphite $(6 \mathrm{~mL}, 24 \mathrm{mmol})$ and the mixture stirred at $35^{\circ} \mathrm{C}$. After $16 \mathrm{~h}$, water $(30 \mathrm{~mL})$ was added and the mixture stirred at ambient temperature further 30 min. and the organic phase was dried $\left(\mathrm{MgSO}_{4}\right)$ and rotavaporated. The oily residue was suspended in silica and chromatographed (vacuum, silica, dichloromethane/ethyl acetate: 10/1), affording compound 4 as colorless oil (5.13 g, 79\%). IR(neat) $v_{\max }=3167,2982$, 2936, 1720, 1252, $995 \mathrm{~cm}^{-1} ;{ }^{1} \mathrm{H}-\mathrm{NMR}\left(\mathrm{CDCl}_{3}\right) \delta=1.22-1.30$ (m, 15H, $\left.5 \mathrm{x} \mathrm{Me}\right), 3.29$ (d, $\left.\left.2 \mathrm{H},{ }^{1} \mathrm{~J}_{\mathrm{PH}}=24.0 \mathrm{~Hz}\right), 4.19-4.29\left(\mathrm{~m}, 2 \mathrm{H}, \mathrm{OCH}_{2}\right), 4.66-4.74(\mathrm{~m}, 2 \mathrm{H}, 2 \times \mathrm{OCH}),\right) ;{ }^{13} \mathrm{C}-\mathrm{NMR}$ $\left(\mathrm{CDCl}_{3}\right)$ : $14.1(\mathrm{Me}), 23.6(\mathrm{Me}), 23.7(\mathrm{Me}), 24.6(\mathrm{Me}), 24.6\left(\mathrm{~d},{ }^{1} J_{\mathrm{PC}}=83.8 \mathrm{~Hz}\right), 61.5$ $\left(\mathrm{OCH}_{2}\right), \sim 71.1\left(\mathrm{~d},{ }^{2} J_{\mathrm{PC}}=13.6 \mathrm{~Hz}, \mathrm{OCH}\right), 143.6(\mathrm{C}=\mathrm{N}), 163.9(\mathrm{CO})$. HRMS (ESI-TOF) calcd 296.1263 [M+1]; found 296.1258.

\section{Synthesis of ethyl 3-diisopropylphosphoryl-2-(4-methylphenylsulfonylimino) propanoate 5}

To a solution of compound $4(4.5 \mathrm{~g}, 15 \mathrm{mmol})$ in dichlorometane $(40 \mathrm{~mL})$ was added sodium carbonate $(4.8 \mathrm{~g}, 45 \mathrm{mmol})$ followed by tosyl chloride $(3.24 \mathrm{~g}, 17 \mathrm{mmol})$ and the mixture stirred until the disappearance of the starting oxime $(\sim 4 \mathrm{~h})$. Filtration off the insolubles and evaporation of the solvent afforded a residue. This, suspended in silica and 
chromatographed (vacuum, silica, hexanes-dichloromethane-ethyl acetate increasing polarity) produced compound $5(4.2 \mathrm{~g}, 62 \%)$ as a pale yellow thick oil. IR(neat) $v_{\max }=$ 2983, 1737, 1386, 1267, 1194, $993 \mathrm{~cm}^{-1} ;{ }^{1} \mathrm{H}-\mathrm{NMR}\left(\mathrm{CDCl}_{3}\right) \delta=1.19-1.30$ (m, 15H, $\left.5 \mathrm{x} \mathrm{Me}\right)$, $2.38(\mathrm{~s}, 3 \mathrm{H}, \mathrm{PhMe}), 3.25\left(\mathrm{~d}, 2 \mathrm{H},{ }^{1} J_{\mathrm{PH}}=24.0 \mathrm{~Hz}\right), 4.23\left(\mathrm{q}, 2 \mathrm{H}, J=6.0 \mathrm{~Hz}, \mathrm{OCH}_{2}\right), 4.57-4.64$ $(\mathrm{m}, 2 \mathrm{H}, 2 \times \mathrm{OCH}), 7.28(\mathrm{~d}, 2 \mathrm{H}, J=6.0 \mathrm{~Hz}), 7.83(\mathrm{~d}, 2 \mathrm{H}, J=6.0 \mathrm{~Hz}) ;{ }^{13} \mathrm{C}-\mathrm{NMR}\left(\mathrm{CDCl}_{3}\right)$ : $13.8(\mathrm{Me}), 21.6(\mathrm{Me}), 23.4(\mathrm{Me}), 23.5(\mathrm{Me}), 23.6(\mathrm{Me}), 23.7(\mathrm{Me}), 26.5\left(\mathrm{~d},{ }^{1} J_{\mathrm{PC}}=135.8\right.$ $\left.\mathrm{Hz}, \mathrm{PCH}_{2}\right), 62.8(\mathrm{OCH}), \sim 72.0\left(\mathrm{~d},{ }^{2} J_{\mathrm{PC}}=12.1 \mathrm{~Hz}, \mathrm{OCH}\right), 131.7(\mathrm{C}, \mathrm{Ar}), 145.7(\mathrm{C}, \mathrm{Ar})$, $152.1(\mathrm{C}=\mathrm{N}), 161.1(\mathrm{CO})$. HRMS (ESI-TOF) calcd 450.1351 [M+1]; found 450.1346.

\section{Synthesis of cycloadducts 7}

\section{General Procedure}

To ethyl 3-(diisopropylphosphoryl)-2-(4-methylphenylsulfonylimino)propanoate 5 (0.3 g; $0.69 \mathrm{mmol})$ in benzene $(5 \mathrm{~mL})$ was added triethylamine ( 0.33 eq.; $30 \mu \mathrm{L}), \mathrm{K}_{2} \mathrm{CO}_{3}$ (10 eq.; $0.96 \mathrm{~g} ; 6.9 \mathrm{mmol}$ ) and the diene (1.0 eq. - large excess). Cyclopentadiene was used in large excess $(1 \mathrm{~mL})$, other dienes were used in excess: Danishefsky diene (1.5 eq), 1methoxy1,3-butadiene (2 eq.), 2,3-dimethyl-1,3-butadiene (1.5 eq. or 5 eq.). The reaction mixtures were stirred for 4 days at rt. Evaporation of the solvent gave the crude that was suspended in silica and chromatographed (vacuum, silica, petroleum ether-ether, polarity gradient). In the case of reaction with Danishefsky diene chromatography was run in a mixture of ethyl acetate and methanol (3:1).

Ethyl 7-(diisopropylphosphoryl)-3,4-dimethyl-1-azabicyclo[4.1.0]hept-3-ene-6carboxylate 7a

i) 2,3-dimethy1,3-butadiene (1.5 eq.). Yield $(0.020 \mathrm{~g}$; $0.056 \mathrm{mmol} ; 8.0 \%)$.

ii) 2,3-dimethy1,3-butadiene (5.0 eq.). Yield ( $0.025 \mathrm{~g} ; 0.070 \mathrm{mmol} ; 9.3 \%)$.

IR $(\mathrm{NaCl}): v=3458,2981,2933,1753 \mathrm{~cm}^{-1} .{ }^{1} \mathrm{H}-\mathrm{NMR}\left(\mathrm{CDCl}_{3}\right): 1.29-1.33(\mathrm{~m}, 15 \mathrm{H}, 5 \mathrm{x}$ Me), 1.52 (br s, 3H, Me), 1.63 (br s, 3H, Me), 2.16 (d, $J=17.3 \mathrm{~Hz}, 1 \mathrm{H}, 7-\mathrm{H}$ ), 2.38 (br d, $J=$ $17.3 \mathrm{~Hz}, 5-\mathrm{H}), 2.70$ (d, $J=17.6 \mathrm{~Hz}, \mathrm{H}-5), 3.23$ (d, $J=17.0 \mathrm{~Hz}, 1 \mathrm{H}, 2-\mathrm{H}), 3.73$ (d, $J=17.0$ $\mathrm{Hz}, 2-\mathrm{H}), 4.16-4.26$ (m, $\left.2 \mathrm{H}, \mathrm{OCH}_{2}\right), 4.68-4.80(\mathrm{~m}, 2 \mathrm{H}, 2 \mathrm{x} \mathrm{OCH}) ;{ }^{13} \mathrm{C}-\mathrm{NMR}\left(\mathrm{CDCl}_{3}\right): 13.1$ (Me), $15.4(\mathrm{Me}), 17.5(\mathrm{Me}), 22.6(\mathrm{Me}), 22.9(\mathrm{Me}), 23.95(\mathrm{Me}), 23.05(\mathrm{Me}), 23.1(\mathrm{Me}), 28.4$ $\left(\mathrm{d},{ }^{3} J_{\mathrm{PC}}=19 \mathrm{~Hz}, \mathrm{CH}_{2}, 5-\mathrm{C}\right),\left(\mathrm{d},{ }^{1} J_{\mathrm{PC}}=216 \mathrm{~Hz}, 7-\mathrm{C}\right), 45.0\left(\mathrm{~d},{ }^{2} J_{\mathrm{PC}}=5.0 \mathrm{~Hz}, \mathrm{C}-6\right), 51.6\left(\mathrm{~d},{ }^{3} \mathrm{~J}\right.$ PC $\left.=6.6 \mathrm{~Hz}, \mathrm{CH}_{2}, 2-\mathrm{C}\right), 60.3\left(\mathrm{CH}_{2} \mathrm{O}\right), 69.8\left(\mathrm{~d},{ }^{3} J_{\mathrm{PC}}=6.0 \mathrm{~Hz}, \mathrm{COH}\right), 69.9\left(\mathrm{~d},{ }^{3} J_{\mathrm{PC}}=6.3 \mathrm{~Hz}\right.$, 
COH), 118.7 (3-C or 4-C), 119.1 (4-C or 3-C), 168.7 (CO). HRMS (FAB) calcd 360.1940 [M+1]; found 360.1927.

Ethyl

7-(diisopropylphosphoryl)-5-methoxy-1-azabicyclo[4.1.0]hept-3-ene-6carboxylate $7 \mathrm{~b}$

Yield $(0.125 \mathrm{~g} ; 0.35 \mathrm{mmol} ; 51 \%)$. IR $(\mathrm{NaCl}): v=3467,2980,2931,1754,1731 \mathrm{~cm}^{-1} .{ }^{1} \mathrm{H}-$ NMR (CDCl $): 1.23-1.28(\mathrm{~m}, 15 \mathrm{H}, 5 \mathrm{x} \mathrm{Me}), 2.30(\mathrm{~d}, J 16.6 \mathrm{~Hz}, 2 \mathrm{H}, 5 \mathrm{H}+7-\mathrm{H}), 2.78(\mathrm{dd}, J$ $=6.1,18.5 \mathrm{~Hz}, 1 \mathrm{H}, 5-\mathrm{H}), 3.61(\mathrm{~s}, 3 \mathrm{H}, \mathrm{OMe}), 4.16-4.18\left(\mathrm{~m}, 2 \mathrm{H}, \mathrm{OCH}_{2}\right), 4.68(\mathrm{br} \mathrm{s}, 2 \mathrm{H}, 2 \mathrm{x}$ OCH), $4.80(\mathrm{~s}, 1 \mathrm{H}, 2-\mathrm{H}), 5.39(\mathrm{~d}, J=10.0 \mathrm{~Hz}, 1 \mathrm{H}, 3-\mathrm{H}), 5.62-5.63(\mathrm{~m}, 1 \mathrm{H}, 4-\mathrm{H}) .{ }^{13} \mathrm{C}-\mathrm{NMR}$ $\left(\mathrm{CDCl}_{3}\right)$ : $\left.14.4(\mathrm{Me}), 24.1(\mathrm{Me}), 24.2 \mathrm{CH}_{2}, 5-\mathrm{C}\right), 24.4(\mathrm{Me}), 24.42(\mathrm{Me}), 24.6(\mathrm{Me}), 33.2(\mathrm{~d}$, $\left.{ }^{1} J_{\mathrm{PC}}=217 \mathrm{~Hz}, 7-\mathrm{C}\right), 45.6\left(\mathrm{~d},{ }^{2} J_{\mathrm{PC}}=4.5 \mathrm{~Hz}, \mathrm{C}-6\right), 57.3(\mathrm{OMe}), 61.8\left(\mathrm{OCH}_{2}\right), 71.3\left(\mathrm{~d},{ }^{2} J_{\mathrm{PC}}\right.$ $=6.8 \mathrm{~Hz}) 71.5\left(\mathrm{~d},{ }^{2} J_{\mathrm{PC}}=6.8 \mathrm{~Hz}, \mathrm{OCH}\right), 85.6(\mathrm{C}-2), 123.1$ (3-C or 4-C), 124.5 (4-C or 3-C), 168.0 (CO). HRMS (FAB) calcd 362.1732 [M+1]; found 362.1731 .

Ethyl 3-(diisopropylphosphoryl)-2-azatricyclo[3.2.1.0 $\left.{ }^{2,4}\right]$ oct-6-ene-4-carboxylate 7d Yield (0.140 g; $0.41 \mathrm{mmol} ; 59.4 \%)$. IR (NaCl): $v=3467,2981,2937,1741 \mathrm{~cm}^{-1} .{ }^{1} \mathrm{H}-\mathrm{NMR}$ $\left(\mathrm{CDCl}_{3}\right): 1.27-1.34(\mathrm{~m}, 15 \mathrm{H}, 5 \mathrm{x} \mathrm{Me}), 1.62(\mathrm{~d}, J=12.5 \mathrm{~Hz}, 3-\mathrm{H}), 1.71(\mathrm{t}, J=8.6 \mathrm{~Hz}, 8-\mathrm{H})$, $2.43(\mathrm{~d}, J=8.6 \mathrm{~Hz}, 1 \mathrm{H}, 8-\mathrm{H}), 3.29(\mathrm{br} \mathrm{s}, 1 \mathrm{H}, 5-\mathrm{H}), 4.24-4.29$ (m, 3H, $\left.\mathrm{OCH}_{2}+1-\mathrm{H}\right), 4.67-$ $4.78(\mathrm{~m}, 2 \mathrm{H}, 2 \times \mathrm{OCH}), 5.71-5.73(\mathrm{~m}, 1 \mathrm{H}, 6-\mathrm{H}$ or $\mathrm{H}-7), 6.18-6.22(\mathrm{~m}, 1 \mathrm{H}, 7-\mathrm{H}$ or $6-\mathrm{H})$; ${ }^{13} \mathrm{C}-\mathrm{NMR}\left(\mathrm{CDCl}_{3}\right): 14.5(\mathrm{Me}), 24.3(\mathrm{Me}), 24.4(\mathrm{Me}), 24.43(\mathrm{Me}), 24.5(\mathrm{Me}), 45.8\left(\mathrm{~d},{ }^{1} J_{\mathrm{PC}}\right.$ $=205 \mathrm{~Hz}, 3-\mathrm{C}), \sim 48(6-\mathrm{C}), 49.6(5-\mathrm{C}), 59.4(\mathrm{~d}, J=3.0 \mathrm{~Hz}, 8-\mathrm{C}), 62.0\left(\mathrm{OCH}_{2}\right), 67.6\left(\mathrm{~d},{ }^{3} J\right.$ $\mathrm{PC}=7.5 \mathrm{~Hz}, 1-\mathrm{C}), 71.3\left(\mathrm{~d},{ }^{2} J_{\mathrm{PC}}=6.8 \mathrm{~Hz}, \mathrm{OCH}\right), 71.5\left(\mathrm{~d},{ }^{2} J_{\mathrm{PC}}=6.8 \mathrm{~Hz}, \mathrm{OCH}\right), 128.8(5-\mathrm{C}$ or 6-C), 133.3 (6-C or 5-C), 172.0 (CO). HRMS (FAB) calcd 344.1627 [M+1]; found 344.1615 .

Ethyl 1-((diisopropylphosphoryl)methyl)-4-oxo-1,2,5,6-tetrahydropyridine-2carboxylate 8

Yield (0.092 g; $0.267 \mathrm{mmol} ; 38.5 \%)$. IR ( NaCl): $v=3440,2983,2938,1733,1633,1573$ $\mathrm{cm}^{-1} .{ }^{1} \mathrm{H}-\mathrm{NMR}\left(\mathrm{CDCl}_{3}\right): 1.21-1.27(\mathrm{~m}, 12 \mathrm{H}, 4 \mathrm{x} \mathrm{Me}), 1.36(\mathrm{t}, J=7.2 \mathrm{~Hz}, 3 \mathrm{H}, \mathrm{Me}), 3.73$ (q, $\left.J=7.2 \mathrm{~Hz}, 2 \mathrm{H}, \mathrm{OCH}_{2}\right), 4.63-4.71(\mathrm{~m}, 2 \mathrm{H}, 2 \mathrm{x} \mathrm{OCH}), 4.71\left(\mathrm{~d}, J=12.0 \mathrm{~Hz}, 2 \mathrm{H}, 1^{\prime}-\mathrm{H}\right), 6.45$ $(\mathrm{dd}, J=3.0,7.6 \mathrm{~Hz}, 5-\mathrm{H}), 7.03(\mathrm{~d}, J=3.0,1 \mathrm{H}, 3-\mathrm{H}), 7.35(\mathrm{~d}, J=7.6 \mathrm{~Hz}, 1 \mathrm{H}, 6-\mathrm{H}) .{ }^{13} \mathrm{C}-$ NMR ( $\mathrm{CDCl}_{3}$ ): $14.3(\mathrm{Me}), 24.21(\mathrm{Me}), 24.27(\mathrm{Me}), 24.32(\mathrm{Me}), 49.7\left(\mathrm{~d},{ }^{1} J_{\mathrm{PC}}=156 \mathrm{~Hz}, 1^{\prime}\right.$ 
-C, $\left.\mathrm{CH}_{2}\right), 63.3\left(\mathrm{CH}_{2} \mathrm{O}\right), 73.0\left(\mathrm{~d},{ }^{2} J_{\mathrm{PC}}=7.5 \mathrm{~Hz}, \mathrm{OCH}\right), 119.7(5-\mathrm{C}), 123.3(3-\mathrm{C}), 140.2$ (2-

C), 162.9 (CO), 179.5 (CO). HRMS (FAB) calcd 346.1419 [M+1]; found 346.1419.

\section{Acknowledgments}

The authors thank Fundação para a Ciência e Tecnologia, CIQA-Centro de Investigação em Química do Algarve and Xunta de Galicia for financial support under project 07CSA008203PR.

\section{References}

[1] For reviews, see: a) F. Palacios, A. M. Ochoa de Retana, E. M. Marigorta, J. M. Santos, Org. Prep. Proced. Int. 2002, 34, 219-269; b) T. L. Gilchrist, Aldrichimica Acta 2001, 34, 51-55; F. Palacios, A. M. Ochoa de Retana, E. M. Marigorta, J. M. Santos, Eur. J. Org. Chem. 2001, 2401-2414.

[2] a) T. M. V. D. Pinho e Melo, C. S. J. Lopes, A. d'A Rocha Gonçalves, J. A. Beja, A. M. Paixão, M. R. Silva, L. Alte da Veiga, J. Org. Chem. 2002, 67, 66-71; b) F. A. Davis, C. Liang, H. Liu, J. Org. Chem. 1997, 62, 3796-3797; c) K. Banet, F. Hohler, Angew. Chem., Int. Ed. 2001, 40, 174-177.

[3] H. Heimgartner, Angew. Chem. , Int. Ed. Eng. 1991, 30, 238-264

[4] T. W. Miller, E. W. Tristram, F. J. Wolf, J. Antibiot. 1971, 24, 48-51.

[5] T. F. Molinski, C. M. Ireland, J. Org. Chem. 1988, 53, 2103-2105

[6] a) D. Tanner, Angew. Chem., Int. Ed. Engl. 1994, 33, 599-619; b) S. N. Filigheddu, M. Taddei, Tetrahedron Lett. 1998, 39, 3857-3860; c) B. Zwanenburg, L. J. Thi, Pure Appl. Chem. 1996, 68, 735-738; d) F. A. Davis, H. Liu, C. V. Reddy, Tetrahedron Lett. 1996, 37, 5473-5476.

[7] a) G. Righi, R. DAchielle, Tetrahedron Lett. 1996, 37, 6893-6896; b) Y. Lim, W. K. Lee, Tetrahedron Lett. 1995, 36, 8431-8434; c) D. Tanner, C. Bergsson, H. K. Dhaliwal, Tetrahedron Lett. 1990, 31, 1903-1906.

[8] M. J. Alves, T. L. Gilchrist, J. Chem. Soc., Perkin 1, 1998, 299-303.

[9] F. A. Davis, Y. Wu, H. Yan, K. R. Prasad, W. McCoull, Org. Lett. 2002, 4, 655-658. 
[10] M. J. Alves, A. Gil Fortes, A. Lemos, C. Martins, Synthesis, 2005, 555-558.

[11] a) V. J. Nair, J. Org. Chem. 1972, 37, 2508-2510; b) A. Hassner, D. J. Anderson, J. Org. Chem., 1974, 39, 2031-2036; c) M. J. Alves, N. G. Azoia,J. F. Bickley, T. L. Gilchrist, R. Mendonça, J. Chem. Soc., Perkin 1, 2001, 2969-2976. d) C. A. Ray, E. Risberg, P. Somfai, Tetrahedron Lett. 2001, 42, 9289-9291. e) C. A. Ray, E. Risberg, P. Somfai, Tetrahedron 2002, 58, 5983-5987.

[12] M. J. Alves, A. Gil Fortes, F. Teixeira Costa, V. C. M. Duarte, Tetrahedron, 2007, 63, 11167-11173.

[13] K. W. Law, L. Ting-Fong, M. P. Sammes, A. R. Katrizky, T. C. W. Mak, J. Chem. Soc., Perkin 1, 1984, 111-118.

[14] K. Isomura, T. Tanaka, T. Tatsuyoshi, H. Taniguchi, Chemistry Letters, 1977, 4, 397 400.

[15] M. J. Alves, unpublished results: 2,2-di-substituted $2 \mathrm{H}$-azirines 3-carboxylates showed no cycloaddition to occur with nucleophilic dienes and cyclopentadiene.

[16] H. Skarpos, V. D. Vorobeva, S. N. Osipov, I. L. Odinets, E. Breuer, G. V. Röschenthaler, Org. Biomol. Chem. 2006, 4, 3669-3674.

[17] R. Engel, In Handbook of Organophosphorus Chemistry; Dekker, M. Inc.: New York, 1992. Phosphorus \& Sulfur, 1991, 63, 193-215.

[18] a) A. B. Young, J. T. Greenamyre, Z. Hollingsworth, R. Albin, C. D'Amato, I. Shoulson, J. B. Penny, Science, 1988, 241, 981-988; b) G. Reyes-Rangel, V. Marañón, C. G. Avila-Ortiz, C. A. Parrodi, L. Quintero, E. Juaristi, Tetrahedron, 2006, 62, 8404-8409.

[19] G. Zhao, U. C. Deo, B. Ganem, Org. Lett. 2001, 3, 201-203.

[20] H. C. J. Ottenheijm, R. Plate, J. H. Noordik, J. D. M. Herscheid, J. Org. Chem., 1982, 47, 2147.

[21] F. Palacios, A. M. Ochoa de Retana, J. I. Gil, J. M. Ezpeleta, J. Org. Chem. 2000, 65, 3213-3217. 